\title{
E-MAC: Efficient MAC Protocol for Time-Critical Transmission in Wireless Sensor Networks
}

\author{
${ }^{* 1}$ Archana R. Raut, ${ }^{2}$ Dr. S. P. Khandait, ${ }^{3}$ Dr. U. N. Shrawankar \\ ${ }^{1}$ Research Scholar, Department of IT, G. H. Raisoni College of Engineering, Nagpur, India \\ ${ }^{2}$ Research Supervisors, Department of IT, G. H. Raisoni College of Engineering, Nagpur, India \\ ${ }^{3}$ Associate Professor, G. H. Raisoni College of Engineering, Nagpur, India \\ Email: archana.kakade5@gmail.com,prapti_khandait@yahoo.co.in,urmila.shrawankar@raisoni.net
}

Received: 09 ${ }^{\text {th }}$ July 2018, Accepted: $14^{\text {th }}$ August 2018, Published: $31^{\text {st }}$ August 2018

\begin{abstract}
Wireless sensor networks (WSNs) are used in numerous ranges of applications to sense the desired data form environment and transmit it to the central coordinator in energy efficient manner in order to increase the network's lifetime. In addition to energy there are few other factors, such as quality of service (QoS) which are also equally essential to get better performance of the WSNs. Industrial control with automation using wireless sensor networks requires extremely reliable and well-timed data delivery. Various medium access control (MAC) protocols and network layer protocols are designed in order to fulfill application based QoS requirements in WSNs terms of transmission delay and reliability. The mission critical WSN applications are sensitive to transmission delays. To defeat this, a very much characterized QoS is important to upgrade WSN flexibility. We proposed E-MAC (Efficient MAC) protocol for time critical data delivery in WSNs.
\end{abstract}

Key Words: Wireless Sensor Network, Quality of Service, Mission-Critical, Delay, Reliability.

\section{Introduction}

Wireless Sensor Network (WSN) is a very important means for closely monitoring and controlling application processes to the end users. The most important reason for establishing the wireless sensor network is to take real-time judgment based on data generated by the sensor nodes (figure 1). This real time transmission of sensor nodes data to the central coordinator is challenging because of the resource constraints and communication capability limitation of sensor nodes. Conventionally, the biggest challenge of sensor network is the restricted lifetime but for real time applications such as military operation, industrial monitoring and control systems, environmental monitoring and health care applications, needs a time bounded and reliable delivery of a data from source node to destination [1-5]. For such applications besides energy, message delivery timeliness is also one of the significant issues. So, based on the type of application it is essential to grant different levels of QoS in WSNs. QoS challenges in WSNs contrast from those in conventional remote systems. The key difficulties identified with WSN are node organization, resource limitations, topology varieties, data redundancy, handling multiple traffic types, real-time traffic and scalability as shown in figure 2, while additional QoS challenges in real time WSN applications are bounded delay, assured throughput, duty cycles and reliable message delivery. In real time applications QoS can be achieved by rising the network throughput and reliability and by reducing the end-to-end delay during data transmission.

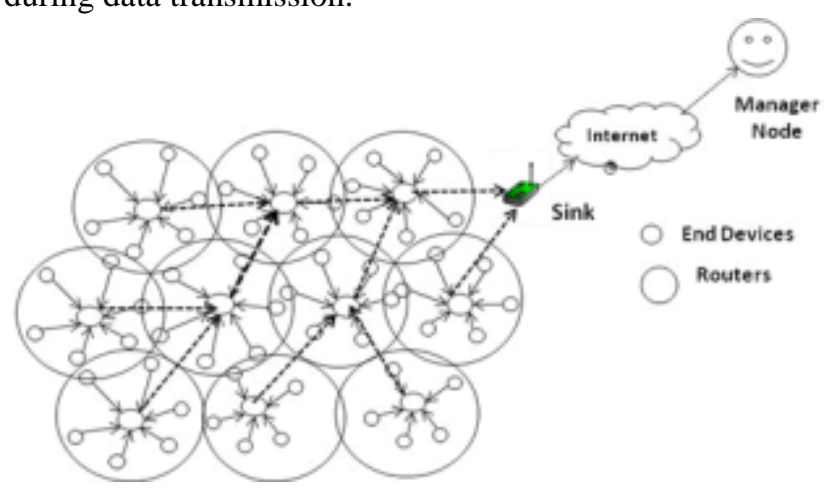

Fig. 1: Wireless Sensor Networks

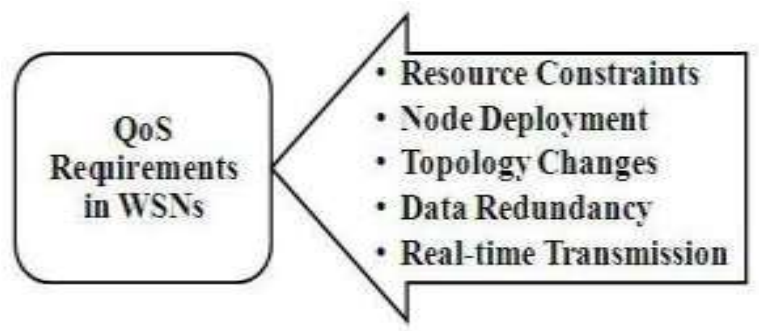

Fig. 2 QoS Challenges in WSNs

\section{Problem Statement:}

WSNs are widely used for variety of real time applications ranging from military surveillance to industrial control and monitoring. To fulfil the needs of industrial control and automation application, QoS requirements must be enhanced to perform better in harsh operating environments. Wireless sensor networks $\mathrm{u} \mathrm{s}$ e $\mathrm{d}$ for industrial process monitoring and control need extremely trustworthy and well-timed data delivery. Various dedicated schedule based medium access control (MAC) protocols are developed [6-7].

In industrial scenario it is essential to determine a schedule that can bear the given application requirements in terms of data delivery latency and reliability. In order to enhance the performance of industrial wireless networks, various protocols are 
developed by researchers. But, in case of time critical applications with harsh deadlines, each data packet including data packet with emergency data has to send without any waiting time to access channel. Also, critical applications are required to deliver critical data in continuous time slots till the complete data is sent to destination. So, to achieve this it is essential to modify QoS based MAC protocols concerning for better energy efficiency, reducing transmission delays, and increasing reliability in order to get higher system throughput.

\section{Related Works}

While expanding RT applications in WSN, resource constraints must be considered in addition to reliability constraints to support desired performance at all time. In recent years, many QoS aware MAC and routing protocols were proposed for WSNs [7-19]. Many of these protocol works for improving energy efficiency, considering energy of sensor nodes as a significant resource. Besides, some additional challenges also caused by time critical applications where both energy proficient and QoS supported routing is required in order to utilize the sensors efficiently and accessing the collected data successfully within time bounds. Figure 3 shows classification of various protocols in view of probabilistic or deterministic approaches.

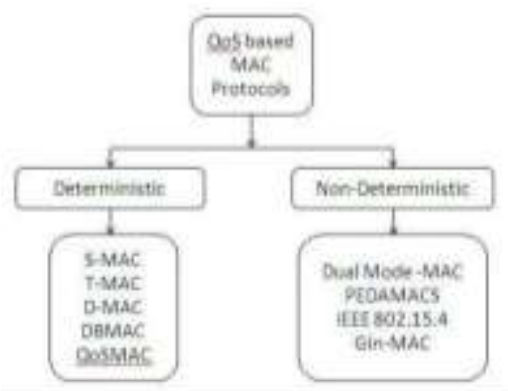

Fig. 3: Classification of QoS based MAC Protocols

In WSNs, main role of the MAC layer is to determine delay required for getting access to the channel, channel utilization and energy requirements. Data transport, reliability and delay are fundamental performance objectives in missioncritical application scenarios [6]. Delay-aware MAC protocols like S-MAC, T-MAC and B-MAC [7-9] etc. only reduce delay to offer best-effort services but RT assurance is not granted. DMAC [10] and DB-MAC [11] protocols are proposed for application-specific data gathering tree but are bounded to use in general topology and even if latency is reduced but precise RT guarantee are not offered by these protocols. In 2006, TDMA-based MAC protocol PEDAMACS [12] is proposed by Ergen, Varaiya to accomplish energy and delay effectiveness. It provides hard RT guarantee but, due to limitation of requirement of powerful AP it cannot be used in variety of applications. IEEE 802.15.4[13] protocol is developed with GTS mechanism for managing time significant data to provide explicit QoS guarantees and is still developing to support hard RT guarantee.
Single-hop hybrid cluster architecture is proposed in 2013 which includes two kinds of nodes transmit only nodes as well as standard nodes $[17,18]$. Here author also proposed a framework for MAC layer protocol called RARE with the purpose of managing the one hop hybrid cluster efficiently and reliably in a self- structured fashion in densely deployed area. This work brings reliable scheduling scheme using transmit-only nodes as well as standard nodes. In [20], BCMN/A protocol also improves network lifetime and reduces network delay by using energy as well as delay efficient data aggregation process both in intra as well as inter-cluster communication. A TDMA-based protocol (DGRAM) that gives significant delay guarantee with desired energy efficiency is proposed in [21]. This protocol is completely self- configuring protocol in which assignment of slots is done with no control messages exchanging. As, it does not provide significant delay guarantee, it is not useful in a wide range of applications.

Presently, only some protocols deal with the dual purpose of attaining delay and reliability bounds metrics, but only some of the proposed protocols can preserve time critical applications. Hence, it is essential to develop event based reliable data transmission methods to support time-critical applications within sensor network domain which gives optimal performance considering QoS guarantee in terms of delay bound and reliability.

\section{Requirements of Industrial WSNs}

In QoS provisioning, MAC layer should provide delay guarantee for getting access to channel whereas network layer should provide the transmission time barrier. Deterministic MAC layer designs are essential to attain low latency in addition to reliable delivery of critical messages to the destination. According to ISA SP 100 working group industrial process control are classified in to following six different classes based on their latency requirement. Table 1 shows applications requirement of each class category with examples.

\begin{tabular}{|l|l|l|}
\hline Class & Applications & $\begin{array}{l}\text { Transmission } \\
\text { Time }\end{array}$ \\
\hline Class 0 & Emergency action & Microseconds \\
\hline Class 1 & $\begin{array}{l}\text { Closed-loop } \\
\text { regulatory control }\end{array}$ & Milliseconds \\
\hline Class 2 & $\begin{array}{l}\text { Closed-loop } \\
\text { supervisory control }\end{array}$ & seconds \\
\hline Class 3 & $\begin{array}{l}\text { Open-loop control } \\
\text { Monitoring with } \\
\text { short-term } \\
\text { operational } \\
\text { consequences }\end{array}$ & Minutes \\
\hline Monitoring without & $\begin{array}{l}\text { immediate } \\
\text { operational } \\
\text { consequences }\end{array}$ & Days \\
\hline
\end{tabular}

Table 1: Class Wise Applications Requirement 
The bio fertilizer $(10 \mathrm{~g} / 1 \mathrm{Kg})$ markedly enhanced the plant shoot length, root length, no of tillers and weight of roots and plant weight in local lake soil when compared with control in paper towel assay.

\section{Proposed System}

The network model used in the research is elaborated as below. In general there are two kinds of networks on the basis of capabilities of nodes; homogenous and heterogeneous. At initial stage of deployment all nodes are considered to be having same energy levels, but, whenever network starts working, energy level of nodes decreases arbitrarily. So, after specific time period all the deployed nodes possess different initial energy levels at different stages. Taking this into consideration, heterogeneous networks are considered in our research work. We separate the network into two kinds of nodes leader nodes (LN) and normal nodes $(\mathrm{NN})$. Here leader nodes are the nodes having high energy level and high transreceiver power whereas normal nodes are the nodes with low energy level and restricted trans-receiver power.

All normal nodes have similar properties during network creation phase. We consider $\mathrm{N}$ numbers of nodes randomly scattered across the region to be monitored. The overall region is again separated into small sub regions which are either square, rectangular or both according to network design requirement. These sub regions formed after sub division are called as clusters. Each cluster consists of n no. of normal nodes and one leader node.

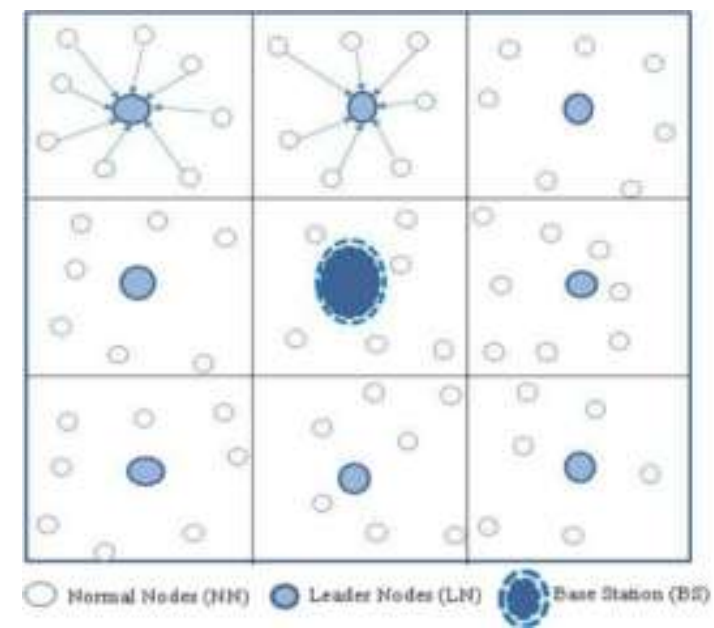

Fig. 4(a): Normal to Leader Nodes Communication

In industrial applications generally a centralized control model is used. Therefore, the star network is considered in each sub-regions i. e. cluster. Each leader node is at one hop distance from the base station (BS). Normal nodes perform the function of data sensing and send it to leader nodes in one hop. Leader none which is at one hop distance from sink node send data to sink node for necessary action. The network topology is shown in Fig. 4 which consists of the normal nodes, leader nodes and the central controller called as base station.

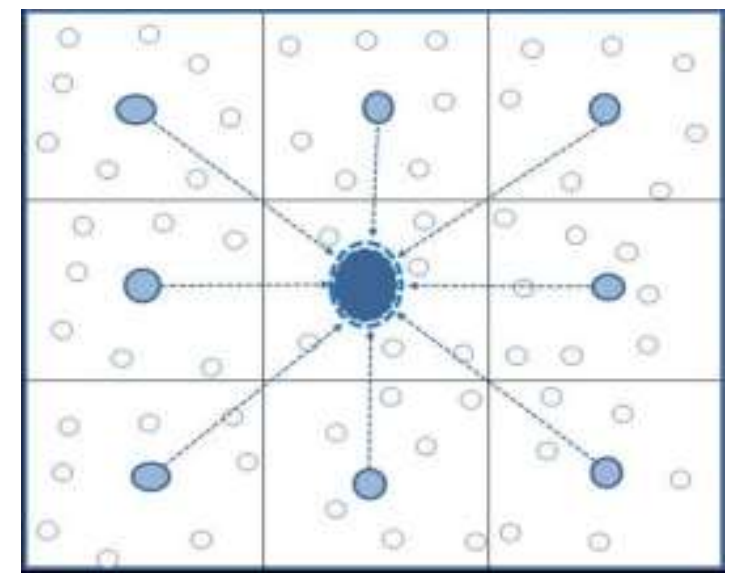

Fig. 4(b): Leader Nodes to Sink Node Communication

\section{Proposed MAC Algorithm}

In the proposed MAC protocol, each TDMA frame is assigned some guaranteed time slots (GTS) for critical data transmission. Also each node is initially assigned with a specific threshold value. With this each node will able to identify channel access priorities which is use to deterministically determine the channel access order for high priority data. This procedure will ensure that in each intercession cycle the high critical data can be the only one to obtain the highest priority, and immediately gain access to the wireless medium.

The essential system in proposed design includes a series of steps after deploying the and they are as per the following,

Step 1- Data sensing by normal sensor nodes.

Each leader node communicates the ping message from its area to identify the nearby nodes located at one hop distance from its location. The ping message informs the leader nodes about its entire one hop neighboring nodes and all normal nodes about their respective leader node. This is practical by communicating ping message every often. The ping message exchanges nodes location to each other and furthermore to illuminate about rest and wake cycles.

\section{Step 2- Data Classification}

The normal nodes will sense desired parameter it is designed for and perform data classification based on the received data and pre-assigned threshold value. Data is classified into three categories as shown in table 2 and then high priority data will be given first priority and sent to respective leader node using assigned arbitrary frequency.

\begin{tabular}{|r|l|l|}
\hline $\begin{array}{r}\text { S. } \\
\text { No. }\end{array}$ & \multicolumn{1}{|c|}{ Data Category } & Priority \\
\hline 1 & Critical (Data value>=Threshold) & High \\
\hline 2 & $\begin{array}{l}\text { Semi-Critical (Data value near } \\
\text { to Threshold) }\end{array}$ & Medium \\
\hline 3 & Normal (Data value<<Threshold) & Low \\
\hline
\end{tabular}

Table 2: Data Classification based on Priority 
Helix Vol. 8(5): 3853- 3857

The decision of a threshold value is based on application scenario. If the readings from the sensor node is with critical data (i.e. data value greater than threshold) are set at high priority. Similarly sensor node with Moderate data (i.e. data value nearly equal to threshold) is set at Medium priority. Whereas, nodes with normal data (i.e. data value very less than threshold) are set at low priority.

Highest priority data will get the immediate channel access using GTS slots. If no GTS slot is available, packets are queued in queue 1 and wait for transmission. To handle moderate data priority queue 2 is maintained. All nodes with semi-critical data maintains Priority queue 2 and if high priority data is not present in queue $1 \mathrm{i}$. e. if queue 1 is empty then priority queue 2 is served. No action is taken for low priority data and only data log is maintained for such data packets at each node level.

Step 3- Based on priority, the node having top priority i.e. priority 1 will get the channel access in s $\mathrm{t}$ a $\mathrm{n} \mathrm{t} 1 \mathrm{y} \mathrm{f}$ o $\mathrm{r}$ data transmission, whereas, low and medium priority nodes will get the channel access after all higher priority nodes will processed. Here the node in high priority loop must be given first priority. Also, whenever user gets channel access it is allocated to user till the complete data transmission is finished. The nodes which are at one hop distance from the sink node can directly send critical data to sink nodes without using cluster head as intermediary.

Step 4- If more nodes are present in the same medium priority queue, then slots are assigned to the nodes one after the other. If there is no node with first priority then only priority queue 2 will be served using regular TDMA scheme.

Step 5- After successful data transmission acknowledge is sent to the sender. However for unsuccessful transmissions No-acknowledge (N-ack) are sent to sender. On receiving $\mathrm{N}$-ack sender has to re-transmit the packet using same procedure.

According to this scheme, first preference is given to the high priority data, second preference to the moderate priority data and only data $\log$ is maintained for third preference i.e. low priority data does not need to send to the base station.

In proposed scheme, high priority data will judge as an intrusion or imposition of critical condition. Sensor node with critical data will be given immediate channel access using assigned arbitrary so that, base station after receiving the messages will take appropriate actions. Thus, with this mechanism we can ensure that the user with highest priority will immediately gain channel access and we can guarantee a deterministic behavior.

\section{Algorithm: \\ SNI Assume all nodes are in idle initial state and channel $\mathrm{N}(\mathrm{t})$}

Step 1: Sensor nodes gather data
Data Classification: Initialize $\mathrm{i}=3 ; \mathrm{j}=2$;

$\mathrm{k}=1$; Classifier $\mathrm{C}=\mathrm{Pr}=\mathrm{k}$ is the highest

priority (sensitive data)

$\mathrm{C}=\mathrm{Pr}=\mathrm{j}$ is the medium priority (semi sensitive data) $\mathrm{C}=\mathrm{Pr}=\mathrm{i}$ is the periodic/low priority data So, $\operatorname{Pr}(\mathrm{i}<\mathrm{j}<\mathrm{k})$

Goto step 2

Step 2:

If $\mathrm{NNi}=$ one hop from sink node AND buffer $\mathrm{N}=\mathrm{Max}$ and Max neighbour node

Then send the data to sink.

Else send the data to LNi. Goto step 3

If $\mathrm{NNj}$ one hope node is $>\mathrm{NNi}$, priority to $\mathrm{NNj}$

Else priority to NNi. Goto step 4 Else goto step 2

End If

End If

Step 3:

The queue processes data for transmission based on the priority level

If $x(E)=1$ event occurs with

data $\mathrm{Q}(\mathbf{0} \ldots \mathrm{I})=\mathrm{N}=\mathrm{MAX}(\mathrm{PrQ} 1)$

Then, Goto to step 4

Else if PrQ1 is empty, Goto to step 4

Else Goto step 5

End If

End If

Step 4: Transmit data to base station;

Wait for Acknowledgement

If (Ack $==1)$ Then Success

Then process the next highest priority

Else process $\operatorname{Pr}(\mathrm{j}<\mathrm{k})$

Else process $\operatorname{Pr}(\mathrm{i}<\mathrm{j})$

Else goto step 5

End If

Step 5: Wait for Non-Acknowledgement (N-

Ack) If $(\mathrm{N}-\mathrm{Ack}==1)$ Then goto step 4

Else goto step 5

End If

\section{Conclusions}

Based on Literature review \& study in, industrial control and automation applications in wireless sensor networks require extremely reliable and welltimed data delivery. We designed a MAC protocol for handling time critical data transmission. The network topology planned uses two hop communications from sensor nodes to base station. The proposed MAC layer protocol is designed for guaranteed channel access for critical data delivery. Proposed MAC protocol is to be tested over existing protocols for performance analysis based on various parameters such as end to end delay and reliability of data transmission. In further work we will implement the proposed protocol to prove its efficiency.

\section{References}

1.Al-Karaki, J.N.; Kamal, A.E. Routing Techniques in Wireless Sensor Networks: A Survey. IEEE Wirel. Commun. 2004, 11, 6-28. 
2. Yanjun Li, Chung Shue Chen, Ye-Qiong Song, Zhi Wang. Real-time QoS support in wireless sensor networks: a survey. 7th IFAC International Conference on Fieldbuses \& Networks in Industrial \& Embedded Systems FeT', Nov 2007, France. 3. T. Arampatzis, J. Lygeros, and S. Manesis, "A survey of applications of wireless sensors and wireless sensor networks," in Proceedings of the 20th IEEE International Symposium on Intelligent Control (ISIC '05), pp. 719-724, June 2005.

4. D. Chen and P. K. Varshney, "QoS Support in Wireless Sensor Network: A Survey," Proceedings of International Conference on Wireless Networks (ICWN), Las Vegas, Nevada, USA, June 2004.

5. Josip Balen, D Zagar, G Martinovic, "Quality of Service in Wireless Sensor Networks: A Survey and Related Patents".

6. W. Ye, J. Heidemann, and D. Estrin,"An energyefficient MAC protocol for wireless sensor networks," in Proc. 21st Annu. Joint Conf. IEEE Computer and Communications Societies, New York, NY, USA, 2002, vol. 3, pp. 1567-1576.

7. T. v. Dam, and K. Langendoen, "An Adaptive Energy-Efficient MAC Protocol for Wireless Sensor Networks," in Proc. 1st ACM Conf.Embedded Networked Sensor Systems, Los Angeles, CA, USA, 2003, pp. 171-180.

8. Polastre, J, J. Hill,D. Culler (2004). Versatile low power media access for wireless sensor networks. In: Proc. ACM Sensys. pp. 95-107.

9. G. Lu, B. Krishnamachari, and C. S. Raghavendra, "An adaptive energy efficient and low-latency MAC for data gathering in wireless sensor networks", in Proc. 18th Int. Parallel and Distributed Processing Symp., Santa Fe, NM, USA, 2004, pp. 224-231.

10. Bacco, G.D., T. Melodia and F. Cuomo (2004). A MAC protocol for delay-bounded applications in wireless sensor networks. In: Proc. Med Hoc-Net. pp. 208-220.

11. S. C. Ergen, P. Varaiya, "PEDAMACS: Power Efficient and Delay Aware Medium Access Protocol for Sensor Networks," IEEE Trans.Mobile Comput., vol.5, pp.920-930, Jul. 2006.

12. Seong-eun Yoo, Poh Kit Chong, Yoonmee

Doh, Minh-Long Pham, Daeyoung Kim, Eunchang Choi, and Jaedoo Huh, "Guaranteeing Real-Time

Services for Industrial Wireless Sensor Networks With IEEE 802.15.4", IEEE Transactions On Industrial Electronics, Vol. 57, No. 11, Pp.38683876, November 2010.

13. Tian Hea, John A Stankovica, C. Lub, T. Abdelzahera, "SPEED: astateless protocol for realtime communication in sensor networks". In:Proc. ICDCS. pp. 46-55.

14.E.Felemban, C.Lee, E Ekici,"MMSPEED: Multipath Multi SPEED protocol for QoS

Guarantee of Reliability and Timeliness in Wireless Sensor Networks," IEEE Trans. Mobile Comput., vol.5, pp.738-754, Jun. 2006.
15. M. Strasser, A. Meier, K. L, and P. Blum, "Dwarf: DelayaWAre Robust Forwarding for Energy-Constrained Wireless Sensor Networks," in Proc. 3rd IEEE Int. Conf. Distributed Computing in Sensor Systems, Santa Fe, USA, 2007, pp. 64-81.

16. HART Communication Foundation, "WirelessHART Technology," [Online]. Available: http://www.hartcomm.org/protocol/wihart/ wireless_technology.html, Dec. 2009.

17. S. Heikalabad, H. Rasouli, F.Nematy,N. Rahmani, "QEMPAR: QoS and Energy Aware

Multi-Path Routing Algorithm for Real-Time Applications in Wireless Sensor Networks", IJCSI International Journal of Computer Science Issues, Vol. 8, Issue 1, January 2011.

18. Jia Zhao, C Qiao, R S. Sudhaakar, Seokhoon Yoon, "Improve Efficiency and Reliability in Single-Hop WSNs with Transmit-Only Nodes", IEEE Transactions On Parallel And Distributed Systems, Vol. 24, No. 3, March 2013.

19. Hady S. AbdelSalam, Stephan Olariu, "Toward

Efficient Task Management in Wireless Sensor Networks", IEEE Transactions On Computers, Vol. 60, No. 11, November 2011.

20. M Dong, K Ota, "Joint Optimization of

Lifetime and Transport Delay under Reliability Constraint Wireless Sensor Networks", IEEE Transactions On Parallel And Distributed Systems, TPDS-2013-12-1250.

21. C Shanti, A Sahoo, "DGRAM: A Delay

Guaranteed Routing and AC Protocol for Wireless Sensor Networks", IEEE Transactions On Mobile Computing, Vol. 9, No. 10, October 2010. 